\title{
A novel likely pathogenic variant in a patient with Hermansky-Pudlak syndrome
}

\author{
Lisa A. Lansdon, ${ }^{1,2,3}$ Dong Chen, ${ }^{4}$ Eric T. Rush, ${ }^{5,6,7}$ Kendra Engleman, ${ }^{5,6}$ \\ Lei Zhang, ${ }^{1,3}$ Carol J. Saunders, ${ }^{1,2,3}$ and Gabor Oroszi ${ }^{1,3}$ \\ ${ }^{1}$ Department of Pathology and Laboratory Medicine, ${ }^{2}$ Center for Pediatric Genomic Medicine, Children's \\ Mercy Kansas City, Kansas City, Missouri 64108, USA; ${ }^{3}$ Department of Pathology and Laboratory Medicine, \\ University of Missouri-Kansas City, School of Medicine, Kansas City, Missouri 64110, USA; ${ }^{4}$ Department of \\ Laboratory Medicine and Pathology, Mayo Clinic, Rochester, Minnesota 55901, USA; ${ }^{5}$ Division of Clinical \\ Genetics, Department of Pediatrics, Children's Mercy Kansas City, Kansas City, Missouri 64108, USA; \\ ${ }^{6}$ Department of Pediatrics, University of Missouri-Kansas City, School of Medicine, Kansas City, Missouri \\ 64110, USA; ${ }^{7}$ Department of Internal Medicine, University of Kansas School of Medicine, Kansas City, Kansas \\ 66160, USA
}

Corresponding author: goroszi@cmh.edu

(C) 2021 Lansdon et al. This article is distributed under the terms of the Creative Commons Attribution-NonCommercial License, which permits reuse and redistribution, except for commercial purposes, provided that the original author and source are credited.

Ontology terms: abnormal number of dense granules; iris transillumination defect

Published by Cold Spring Harbor Laboratory Press

doi:10.1101/mcs.a006110
Abstract Hermansky-Pudlak syndrome (HPS) is a genetic disorder characterized by oculocutaneous albinism and variable pulmonary fibrosis, granulomatous colitis, or immunodeficiency. The diagnosis relies on clinical findings, platelet transmission electron microscopy studies showing absent dense granules, or the identification of a pathogenic genotype in one of 11 associated genes, including HPS1. We report a 2-wk-old male with significant iris transillumination defects, a pale fundus, and mild corectopia found by clinical exome sequencing to have a previously reported pathogenic variant, c.972dupC p.(Met325HisfsTer128), and a variant of uncertain significance, c.1846G >A p.(Glu616Lys), in HPS1. To determine whether his phenotype was consistent with HPS, follow-up studies of whole blood lumiaggregometry and platelet transmission electron microscopy were performed that revealed absent or markedly reduced platelet ATP secretion and virtually absent platelet dense granules, thus confirming the diagnosis. To the best of our knowledge, our case is the first in which the c.1846G>A p.(Glu616Lys) variant is identified in a patient with HPS. In addition, the case also highlights the importance of leveraging appropriate confirmatory clinical testing and reverse phenotyping, which allowed the care team to establish the clinical diagnosis of HPS and reclassify the previously reported variant of uncertain significance in HPS1 to likely pathogenic.

[Supplemental material is available for this article.]

\section{INTRODUCTION}

Hermansky-Pudlak syndrome (HPS; MIM: 203300) is a rare disorder characterized by oculocutaneous albinism, a bleeding diathesis, and variable penetrance of pulmonary fibrosis, granulomatous colitis, and immunodeficiency (Huizing et al. 1993; Oh et al. 1996). Specific ocular findings often include iris and retinal hypopigmentation with iris transillumination, foveal hypoplasia with significant reduction in visual acuity, nystagmus, and increased crossing of the optic nerves. In addition, the skin pigmentation of affected individuals is commonly lighter than that of other family members, and hair color ranges from white to brown. The presence of oculocutaneous albinism and absent delta granules 
COLD SPRING HARBOR Molecular Case Studies
A novel likely pathogenic variant in HPS1 upon platelet transmission electron microscopy studies is sufficient for the clinical diagnosis of HPS (Witkop et al. 1987; Huizing et al. 1993).

Biallelic variants in one of 11 genes (AP3B1, AP3D1, BLOC1S3, BLOC1S5, BLOC1S6, DTNBP1, HPS1, HPS3, HPS4, HPS5, or HPS6) are associated with HPS, with missense or nonsense variants in HPS1 accounting for $37.5 \%$ of cases (Huizing et al. 1993). Founder variants in individuals of Puerto Rican descent have been identified in HPS1 and HPS3 and account for $\sim 78 \%$ and $22 \%$ of cases in that population, respectively (Huizing et al. 1993).

Here, we report a patient with compound heterozygous variants in HPS1 in whom additional studies, including whole blood lumiaggregometry (at 6 mo of age) and platelet transmission electron microscopy (at 8 mo of age), were needed to characterize an identified variant of uncertain significance and confirm the diagnosis.

\section{RESULTS}

\section{Case Report}

The patient, a now 12-mo-old male of European descent with light brown hair and type II skin (defined as "fair" by the Fitzpatrick Scale), was born to a 28-yr-old G1P0 mother by vaginal delivery at $38 \mathrm{wk}$ gestational age. He weighed $3125 \mathrm{~g}$ (32nd centile) and had a length of $48 \mathrm{~cm}$ (16th centile). The pregnancy was uneventful, with normal ultrasound examination and fetal movements. During circumcision he was felt to have mild hypospadias, and the procedure was stopped with referral to pediatric urology. His immediate postnatal course was largely benign, and he was discharged on the second day of life. Shortly after discharge, his parents noticed right corectopia, which was subsequently evaluated by a pediatric ophthalmologist with findings of iris transillumination defect and pale fundus. Specific concern was raised for anterior segment dysgenesis or ocular albinism. Other than appreciation of the right corectopia, the patient was not observed to be dysmorphic on examination. At 2 mo of age the patient began having rust-streaked emesis. He did not have any unusual bruising, epistaxis, mucosal bleeding, hematuria, or hematochezia. He has experienced no feeding difficulty and his growth has been normal. At the time of his most recent visit (8 mo of age), his length was $69 \mathrm{~cm}$ (23rd centile) and his weight was $8.78 \mathrm{~kg}$ (55th centile), and evaluation under anesthesia showed phimosis and orthotopic urethral meatus. His development has been normal thus far. Parents expressed concern over several episodes of abnormal movement, but these were evaluated by a neurology consultant and thought to be nonepileptiform. Microarray analysis was ordered, which was negative for clinically significant findings (see Methods). The patient has experienced no further bleeding episodes and is currently clinically well.

\section{Exome Sequencing}

Trio clinical exome sequencing was performed, and two variants were identified in trans in HPS1 (10q24.2): a paternally inherited pathogenic variant, c.972dupC p.(Met325HisfsTer128); and a maternally inherited variant of uncertain significance (VUS), c.1846G>A p.(Glu616Lys) (Table 1).

\section{Functional Analyses}

To determine whether this patient has a definite diagnosis of HPS, platelet aggregation and secretion studies at Children's Mercy Hospital and platelet transmission electron microscopy at Mayo Clinic Laboratories (see Methods) were undertaken. 


\begin{tabular}{|c|c|c|c|c|c|c|c|c|c|}
\hline Gene & Chromosome & $\begin{array}{c}\text { HGVS } \\
\text { DNA } \\
\text { reference }\end{array}$ & $\begin{array}{l}\text { HGVS protein } \\
\text { reference }\end{array}$ & $\begin{array}{l}\text { Variant } \\
\text { type }\end{array}$ & $\begin{array}{l}\text { Predicted } \\
\text { effect }\end{array}$ & $\begin{array}{l}\text { dbSNP/ } \\
\text { dbVar ID }\end{array}$ & Genotype & ClinVar ID & $\begin{array}{l}\text { Parent } \\
\text { of } \\
\text { origin }\end{array}$ \\
\hline HPS1 (NM_000195.3) & $\begin{array}{c}\text { 10:100186986- } \\
100186987\end{array}$ & c.972dupC & p.(Met325HisfsTer128) & Frameshift & $\begin{array}{l}\text { Loss of } \\
\text { function }\end{array}$ & rs281865083 & Heterozygous & VCV000005278.10 & Paternal \\
\hline HPS1 (NM_000195.3) & 10:100179813 & c. $1846 \mathrm{G}>\mathrm{A}$ & p.(Glu616Lys) & Missense & Substitution & rs775570414 & Heterozygous & VCV000879090.2 & Maternal \\
\hline
\end{tabular}

\section{Impedance Aggregation with Simultaneous ATP Release}

Whole blood lumiaggregometry revealed absent or markedly reduced ATP secretion along with normal maximal aggregation in response to the agonists tested as detailed in Table 2. Platelet count was normal $(302.000 / \mu \mathrm{L})$ at the time of testing and the daily normal quality control sample showed normal secretion with all agonists tested. These findings were suggestive of inherited storage pool disorder (granule deficiency vs. secretion defect), if effects of medications could be ruled out.

\section{Platelet Transmission Electron Microscopy (PTEM)}

Whole mount (WM) and thin section (TS) platelet TEM studies were performed as previously described (Chen et al. 2018). WM-PTEM demonstrated virtually absent platelet-dense granules (see Fig. 1). TS-PTEM was normal, and no alpha granule deficiency or abnormal inclusions were identified. Supplemental testing by buffy coat preparation for evaluation of leukocyte morphology was within normal limits, and no abnormal membranous inclusions or other abnormalities were noted.

These studies revealed severe platelet dense granule deficiency that, in combination with the oculocutaneous albinism observed in our patient, was sufficient to result in the definitive diagnosis of HPS.

\section{DISCUSSION}

HPS is an autosomal recessive, multisystem disorder in which oculocutaneous albinism, bleeding diathesis, and lysosomal ceroid storage result from defects of multiple cytoplasmic organelles: melanosomes, platelet-dense granules, and lysosomes (MIM: 203300). It is most

Table 2. Summary of platelet whole blood lumiaggregometry

\begin{tabular}{|c|c|c|c|c|}
\hline \multirow[b]{2}{*}{ Agonist (concentration) } & \multicolumn{2}{|c|}{ Reference range } & \multicolumn{2}{|c|}{ Patient result } \\
\hline & $\begin{array}{l}\text { Aggregation } \\
\text { (ohm) }\end{array}$ & $\begin{array}{l}\text { Secretion } \\
(\mathrm{nmol})\end{array}$ & $\begin{array}{l}\text { Aggregation } \\
\text { (ohm) }\end{array}$ & $\begin{array}{l}\text { Secretion } \\
\text { (nmol) }\end{array}$ \\
\hline ADP $(10 \mu \mathrm{M})$ & $\geq 6$ & $\geq 0.25$ & 14 & 0.00 \\
\hline $\begin{array}{l}\text { Arachidonic acid } \\
(0.5 \mathrm{mM})\end{array}$ & $\geq 9$ & $\geq 0.50$ & 16 & 0.00 \\
\hline Collagen $(5 \mu \mathrm{g} / \mathrm{mL})$ & $\geq 9$ & $\geq 0.67$ & 10 & 0.00 \\
\hline Collagen $(1 \mu \mathrm{g} / \mathrm{mL})$ & $\geq 8$ & $\geq 0.40$ & 16 & 0.00 \\
\hline Ristocetin (1 mg/mL) & $>5$ & $\mathrm{~N} / \mathrm{A}$ & 14 & N/A \\
\hline Ristocetin $(0.25 \mathrm{mg} / \mathrm{mL})$ & 0 & $\mathrm{~N} / \mathrm{A}$ & 0 & N/A \\
\hline Thrombin (1 U/mL) & $\mathrm{N} / \mathrm{A}$ & $\geq 0.62$ & $N / A$ & 0.40 \\
\hline
\end{tabular}


COLD SPRING HARBOR Molecular Case Studies
A novel likely pathogenic variant in HPS1
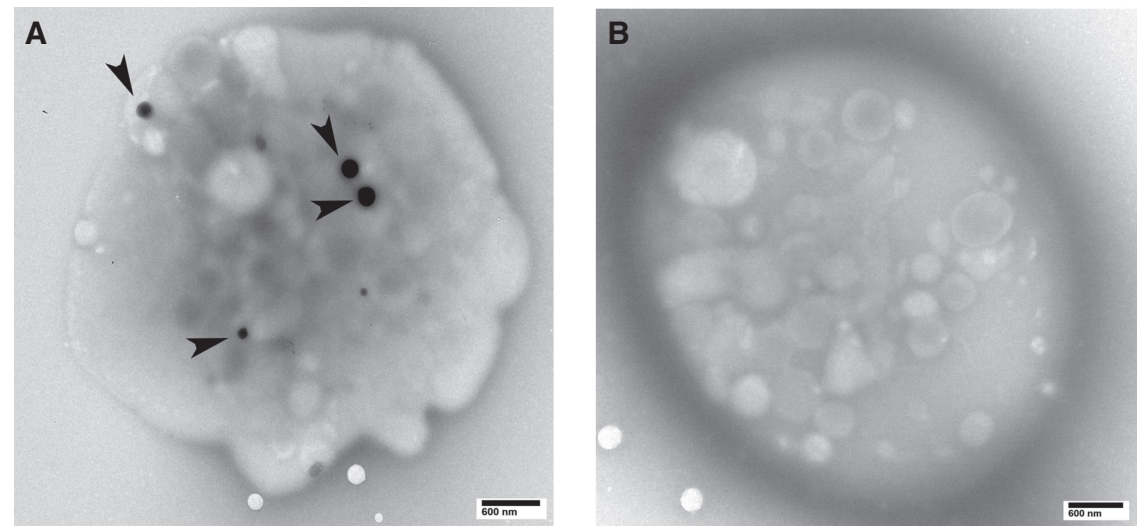

Figure 1. Platelet whole-mount transmission electron microscopy (WM-PTEM). (A) WM-PTEM image of a platelet from a healthy donor. Arrows point to dense granules. (B) WM-PTEM image of a platelet from the patient.

frequently suspected after the observation of clinically significant bleeding in a patient with hypopigmentation and is confirmed upon identification of biallelic pathogenic variants in an HPS-associated gene or the absence of dense granules upon electron microscopy (Huizing et al. 1993, 2020).

Because of the nonspecific nature of our patient's findings, the differential diagnoses were broad and included Wolf-Hirschorn (MIM: 194190), Axenfeld-Rieger (MIM: 180500), Alagille (MIM: 118450), Chediak-Higashi (MIM: 214500), or Hermansky-Pudlak syndromes (HPS; MIM: 203300), other ocular albinism, or congenital aniridia. Thus, chromosomal microarray followed by exome sequencing was pursued, revealing two sequence variants in HPS1 which were classified in accordance with the American College of Medical Genetics and Genomics (ACMG) and Association for Molecular Pathology (AMP) standards and guidelines for the interpretation of sequence variants (Richards et al. 2015).

The paternally inherited c.972dupC p.(Met325HisfsTer128) variant is a frameshift, expected to result in a premature termination codon, causing loss of function due to a truncated or absent protein product due to nonsense-mediated decay. This is a known mechanism of disease for HPS1 and thus is expected to be disease-causing (fulfilling ACMG/AMP "very strong evidence of pathogenicity" criterion PVS1; Richards et al. 2015; Huizing et al. 2020). This variant has been reported in the homozygous state or in trans with a second pathogenic HPS1 variant in multiple individuals affected with HPS ("moderate evidence of pathogenicity" criterion PM3; Huizing et al. 1993, 2020; Oh et al. 1996, 1998; Okamura et al. 2019). In addition, it segregated with HPS in a large Swiss pedigree and is considered to be a Swiss founder variant (Schallreuter et al. 1993). This variant is present in 10 individuals in the gnomAD control data set (10/59786 alleles; 0.002\%) ("moderate evidence of pathogenicity" criterion PM2; Karczewski et al. 2020) and has been reported previously in ClinVar by multiple groups (VCV000005278.8). Therefore, this variant was classified as pathogenic (via one "very strong" [PVS1] and two "moderate" [PM2, PM3] criteria; Richards et al. 2015).

The maternally inherited c.1846G>A p.(Glu616Lys) missense variant substitutes a glutamic acid residue with a lysine residue at position 616. This variant has not been previously reported in affected individuals and is present at a low frequency in the gnomAD control data set (PM2 [7/109402 alleles; 0.006\%]; Karczewski et al. 2020). In silico tools SIFT and PolyPhen-2 predict this variant to be deleterious or possibly damaging, respectively, and this variant resulted in a Combined Annotation Dependent Depletion (CADD) score of 
COLD SPRING HARBOR Molecular Case Studies
A novel likely pathogenic variant in HPS1
27.2 , which predicts that this variant is between the $0.1 \%$ and the $1 \%$ most deleterious changes that can occur in the human genome ("supporting evidence of pathogenicity" criterion PP3; Adzhubei et al. 2010; Sim et al. 2012; Rentzsch et al. 2021). In addition, this variant was previously reported in ClinVar as a VUS (VCV000879090.1), identified in the heterozygous state in a healthy individual as part of a predisposition screen. The presence of this variant in trans with a pathogenic variant was suggestive of clinical significance given the patient's clinical features (PM3), but in the absence of further evidence it was classified as a VUS (via two "moderate" [PM2, PM3] and one "supporting" [PP3] criteria; Richards et al. 2015) and additional studies were recommended.

In preparation for a hypospadias repair, platelet aggregation and secretion studies were performed for the proband, which revealed absent or markedly decreased ATP secretion in the presence of a normal platelet count and normal maximal aggregation. Because of the highly suggestive nature of these findings for platelet storage pool disorder and HPS, confirmatory whole mount EM was ordered. This demonstrated virtually absent dense granules, which is considered a hallmark of HPS, therefore confirming the clinical diagnosis.

Our case illustrates the value of confirmatory clinical testing to establish the correct diagnosis. The young age of our patient and relatively nonspecific phenotypic findings resulted in broad differential diagnoses that were best refined via microarray and exome analyses. These tests allowed for the identification of a highly suggestive HPS1 genotype prompting confirmatory studies showing virtually absent dense granules upon EM. This resulted in a clinical diagnosis of HPS for our patient and allowed for the use of the ACMG/AMP "supporting evidence of pathogenicity" criterion PP4 to reclassify the VUS, c.1846G $>A$ (p.Glu616Lys), to likely pathogenic (via two "moderate" [PM2, PM3] and two "supporting" [PP3, PP4] criteria; Richards et al. 2015). It is possible that this variant will be further reclassified as pathogenic when corroborated by other laboratories.

Of note, at this time we cannot rule out the presence of a deleterious deep intronic variant which would have gone undetected via the genetic analyses used. However, the presence of the variants in trans, their rarity in the general population, and the absence of other reportable coding or splice-site sequence variants in our patient suggest these two variants are responsible for the patient's diagnosis of HPS.

In summary, we successfully diagnosed HPS in an infant who presented with relatively nonspecific phenotypic features via confirmatory clinical testing and reclassified a VUS in HPS1 as likely pathogenic. This study supports the use of both genetic and functional assays in determining patient diagnoses and the importance of leveraging all available resources to resolve VUS if possible.

\section{METHODS}

\section{Genetic Methods}

Genomic DNA was extracted from peripheral blood by a chemagen robot (PerkinElmer) following informed consent. Clinical microarray analysis was performed using the CytoScan HD platform, in accordance with manufacturer recommendations. Analysis was performed using Chromosome Analysis Suite (ChAS 3.3; Thermo Fisher Scientific) to detect copy number variants and absence of heterozygosity. DNA for clinical exome sequencing was prepared using Kapa library prep with IDT i7 exome panel and custom mitochondrial probe enrichment. Next-generation sequencing was performed on an Illumina NovaSeq 6000, with 9.13 Gb of $2 \times 150$ sequenced paired end reads obtained for a mean of $83 \times$ coverage (coverage resulting from clinical exome sequencing of the maternal and paternal samples can be found in Supplemental Table S1). Assembly and alignment of bidirectional sequence to human reference gene sequences based on build GRCh37/UCSC hg19 was performed, and 
custom-developed software, RUNES and VIKING, were used for sequence data analysis. Variants were prioritized and filtered according to variant type, ACMG categorization, OMIM (http://www.omim.org) phenotype, frequency within Children's Mercy's internal variant database (warehouse.cmh.edu), the gnomAD data set (gnomad.broadinstitute.org), and phenotypic concordance with the currently published literature. The Integrative Genomic Viewer software version 2.3.94 (IGV; Broad Institute) was used to visualize alignments, and all variants were interpreted in accordance with the ACMG constitutional sequence variant guidelines (Richards et al. 2015).

\section{Platelet Whole Blood Impedance Lumiaggregometry}

Per manufacturer's recommendations, blood was collected in 2.7-mL blue-top tubes containing 3.2\% sodium citrate as an anticoagulant. Platelet aggregation and secretion studies (as applicable per agonist) were performed in whole blood (WB) diluted in a 1:1 ratio with $0.9 \%$ saline $(450 \mu \mathrm{l}$ or $500 \mu \mathrm{l}$ each) on a Chrono-log Model 700 Lumiaggregometer (Chrono-log Corporation). With each agonist, a diluted WB aliquot was incubated for 5 min at $37^{\circ} \mathrm{C}$ in a plastic cuvette with a magnetic stir bar prior to testing. In the WB impedance method, an AC voltage in millivolt range is applied to the circuit. A stable resistance value is created during the phase of equilibration, when a monolayer of platelets covers the wires. After addition of an agonist, more platelets aggregate on these wires, thereby increasing the resistance to the flow of electric current in the circuit. The change in resistance (maximal amplitude) is reported in ohms. Platelet ATP secretion was measured based on a sensitive luminescent (luciferin-luciferase) assay and the total ATP release with each agonist (as applicable) was reported in nanomoles. For further details of the methodology please see (Zielinski et al. 2018). The final concentration of each agonist is given in Table 2.

\section{Platelet Transmission Electron Microscopy}

As previously described (Chen et al. 2018), whole blood from the patient was collected in acid citrate dextrose (ACD)-A tube and stored at room temperature (RT). Platelet-rich plasma (PRP) was separated from whole blood by centrifugation at $200 \mathrm{~g}$ for $20 \mathrm{~min}$ at RT and then incubated for $30 \mathrm{~min}$ at $37^{\circ} \mathrm{C}$. To perform the WM-PTEM, small drops of platelet rich plasma (PRP) were placed on 300 mesh, formvar-coated, and carbon-stabilized grids (Electron Microscopy Science, Inc), and the whole mount platelet preparation was reviewed using Joel 1200 Exll transmission electron microscope (JEOL Ltd). TS-PTEM was performed by fixing platelets in PRP with glutaraldehyde in Tyrode's buffer (Sigma-Aldrich) at RT. After $20 \mathrm{~min}$, the platelets were centrifuged to pellets that were embedded in 1\% agar (SigmaAldrich). All samples were dehydrated in a graded series of alcohol, subjected to a transmission electron microscopy microwave sample processor (Leica), and embedded in Pure Epoxy resin (Electron Microscopy Science, Inc). Thin sections cut from the plastic blocks on an ultramicrotome were examined on a Joel 1200 Exll transmission electron microscope after staining with uranyl acetate and lead citrate to enhance contrast (Sigma-Aldrich). The method of the buffy coat (white blood cell) TEM is similar to that of the TS-PTEM.

\section{ADDITIONAL INFORMATION}

\section{Data Deposition and Access}

Consent was not given to make these data publicly available. The interpretation of the HPS1 variants detected in our patient were added to their respective entries in ClinVar (https:// www.ncbi.nlm.nih.gov/clinvar/) under accession numbers VCV000005278.10 and VCV000879090.2. 
Competing Interest Statement

The authors have declared no competing interest.

\section{Referees}

Sander Pajusalu

Anonymous

Received May 28, 2021; accepted in revised form July 28, 2021.

\section{Ethics Statement}

Written informed clinical and research consent was obtained for the patient and his parents in compliance with the International Review Board (IRB) at Children's Mercy Kansas City.

\section{Acknowledgments}

We thank the patient and his family for their participation in this study.

\section{Funding}

This work received institutional support from Children's Mercy Kansas City.

\section{REFERENCES}

Adzhubei IA, Schmidt S, Peshkin L, Ramensky VE, Gerasimova A, Bork P, Kondrashov AS, Sunyaev SR. 2010. A method and server for predicting damaging missense mutations. Nat Methods 7: 248-249. doi:10.1038/ nmeth0410-248

Chen D, Uhl CB, Bryant SC, Krumwiede M, Barness RL, Olson MC, Gossman SC, Erdogan Damgard S, Gamb SI, Cummins LA, et al. 2018. Diagnostic laboratory standardization and validation of platelet transmission electron microscopy. Platelets 29: 574-582. doi:10.1080/09537104.2018.1476682

Huizing M, Malicdan MCV, Gochuico BR, Gahl WA. 1993. Hermansky-Pudlak syndrome. In GeneReviews ${ }^{\circledR}$ (ed. Adam MP, Ardinger HH, Pagon RA, et al.). University of Washington, Seattle.

Huizing M, Malicdan MCV, Wang JA, Pri-Chen H, Hess RA, Fischer R, O'Brien KJ, Merideth MA, Gahl WA, Gochuico BR. 2020. Hermansky-Pudlak syndrome: mutation update. Hum Mutat 41: 543-580. doi:10 $.1002 /$ humu.23968

Karczewski KJ, Francioli LC, Tiao G, Cummings BB, Alfoldi J, Wang Q, Collins RL, Laricchia KM, Ganna A Birnbaum DP, et al. 2020. The mutational constraint spectrum quantified from variation in 141,456 humans. Nature 581: 434-443. doi:10.1038/s41586-020-2308-7

Oh J, Bailin T, Fukai K, Feng GH, Ho L, Mao Jl, Frenk E, Tamura N, Spritz RA. 1996. Positional cloning of a gene for Hermansky-Pudlak syndrome, a disorder of cytoplasmic organelles. Nat Genet 14: 300-306. doi:10 .1038/ng1196-300

Oh J, Ho L, Ala-Mello S, Amato D, Armstrong L, Bellucci S, Carakushansky G, Ellis JP, Fong CT, Green JS, et al. 1998. Mutation analysis of patients with Hermansky-Pudlak syndrome: a frameshift hot spot in the HPS gene and apparent locus heterogeneity. Am J Hum Genet 62: 593-598. doi:10.1086/301757

Okamura K, Hayashi M, Abe Y, Kono M, Nakajima K, Aoyama Y, Nishigori C, Ishimoto H, Ishimatsu Y, Nakajima $\mathrm{M}$, et al. 2019. NGS-based targeted resequencing identified rare subtypes of albinism: providing accurate molecular diagnosis for Japanese patients with albinism. Pigment Cell Melanoma Res 32: 848-853. doi:10 1111/pcmr.12800

Rentzsch P, Schubach M, Shendure J, Kircher M. 2021. CADD-Splice-improving genome-wide variant effect prediction using deep learning-derived splice scores. Genome Med 13: 31. doi:10.1186/s13073-021 00835-9

Richards S, Aziz N, Bale S, Bick D, Das S, Gastier-Foster J, Grody WW, Hegde M, Lyon E, Spector E, et al. 2015. Standards and guidelines for the interpretation of sequence variants: a joint consensus recommendation of the American College of Medical Genetics and Genomics and the Association for Molecular Pathology. Genet Med 17: 405-424. doi:10.1038/gim.2015.30

Schallreuter KU, Frenk E, Wolfe LS, Witkop CJ, Wood JM. 1993. Hermansky-Pudlak syndrome in a Swiss population. Dermatology 187: 248-256. doi:10.1159/000247258

Sim NL, Kumar P, Hu J, Henikoff S, Schneider G, Ng PC. 2012. SIFT web server: predicting effects of amino acid substitutions on proteins. Nucleic Acids Res 40: W452-W457. doi:10.1093/nar/gks539

Witkop CJ, Krumwiede M, Sedano H, White JG. 1987. Reliability of absent platelet dense bodies as a diagnostic criterion for Hermansky-Pudlak syndrome. Am J Hematol 26: 305-311. doi:10.1002/ajh 2830260403

Zielinski MD, Stubbs JR, Polites SF, Xue A, Haugen DAL, Emery R, Jenkins DH, Park MS. 2018. In vitro analysis of the hemostatic properties of whole blood products prepared with a platelet-sparing leukoreduction filter. J Thromb Circ Open Access 4: 1. 


\section{COLD SPRING HARBOR Molecular Case Studies}

\section{A novel likely pathogenic variant in a patient with Hermansky- Pudlak syndrome}

Lisa A. Lansdon, Dong Chen, Eric T. Rush, et al.

Cold Spring Harb Mol Case Stud 2021, 7: a006110 originally published online August 6, 2021

Access the most recent version at doi: $10.1101 / \mathrm{mcs} .0006110$
Supplementary http://molecularcasestudies.cshlp.org/content/suppl/2021/08/06/mcs.a006110.D Material C1
License This article is distributed under the terms of the Creative Commons Attribution-NonCommercial License, which permits reuse and redistribution, except for commercial purposes, provided that the original author and source are credited.
Email Alerting Receive free email alerts when new articles cite this article - sign up in the box at the Service top right corner of the article or click here.

